\title{
AVALIAÇÃO DE PARÂMETROS FISIOLÓGICOS DE OVINOS DORPER, SANTA INÊS E SEUS MESTIÇOS PERANTE CONDIÇÕES CLIMÁTICAS DO TRÓPICO SEMI-ÁRIDO NORDESTINO ${ }^{1}$
}

\author{
Physiologic parameters of the Dorper and Santa Inês sheep and \\ their cross submitted to the climatic conditions of the tropic semi-arid northeasterner \\ Marcilio Fontes Cezar ${ }^{2}$, Bonifácio Benício de Souza ${ }^{3}$, Wandrick Hauss de Souza ${ }^{4}$, \\ Edgard Cavalcanti Pimenta Filho ${ }^{5}$, Gustavo de Paula Tavares ${ }^{6}$, Gildenor Xavier Medeiros ${ }^{3}$
}

\begin{abstract}
RESUMO
Com este trabalho teve-se como objetivo avaliar os parâmetros fisiológicos (frequiência respiratória, frequiência cardíaca, movimentos ruminais e temperatura retal) de ovinos das raças Dorper, Santa Inês e seus mestiços $\mathrm{F}_{1}$, submetidos às condições climáticas do trópico semi-árido nordestino. Foram utilizados 48 ovinos, sendo 16 animais de cada um dos três grupos genéticos (Dorper, Santa Inês e mestiço $F_{1}$ Dorper x Santa Inês), dos quais 8 do sexo masculino e 8 do sexo feminino. Utilizou-se o delineamento experimental inteiramente casualizado num esquema fatorial de 3 × 2 × 2 ( 3 grupos de animais, 2 sexos e 2 turnos de coletas). Das três fontes de variações consideradas: sexo, genótipo e turno, a última exerceu efeito significativo $(\mathrm{P}<0,05) \mathrm{em}$ todos os parâmetros fisiológicos analisados, sem interagir com sexo e genótipo, enquanto o genótipo interagiu com o sexo $(\mathrm{P}<0,05)$ em relação à frequiência cardíaca e à respiratória. Concluiu-se que o turno foi o fator que mais influenciou nas respostas dos ovinos ao ambiente térmico, independentemente de genótipo e sexo. O turno da tarde conduziu os animais à situação de perigo, em termos de desconforto térmico, e à condição de elevado estresse calórico. Para a freqüência cardíaca, os machos F1 e as fêmeas Dorper e para frequiência respiratória, os machos e fêmeas Dorper e os machos F1 foram os mais estressados, o que demonstra menor adaptabilidade do genótipo exótico e de seus mestiços.
\end{abstract}

Termos para indexação: Adaptabilidade, estresse térmico, raça, frequiência respiratória, temperatura retal.

\section{ABSTRACT}

The goal of this work was to evaluate physiological parameters (respiratory and cardiac frequencies, rumen movement and rectal temperature) in Dorper, Santa Inês and Dorper x Santa Inês crossbreed (F1) sheep submitted to typical weather conditions (tropic semi-arid) of Brazilian northwest. Were used 48 sheep, 16 animals from each of the three genetic groups (Dorper, Santa Inês and crossbreed), among them 8 were male and 8 were female. The experimental design was completely randomized in a $3 \times 2 \times 2$ factorial scheme ( 3 genetic groups, 2 genders and 2 collecting shift). From the three variations sources considered, gender, genotype and shift, the last had a significant $(\mathrm{P}<0.05)$ effect on all physiological parameters evaluated without interaction with gender $(\mathrm{P}<0.05)$ in relation to respiratory and cardiac frequencies. It was concluded that the shift was the factor that had the higher influence on the sheep's answer to temperature environment, independently of genotype and gender. Evening shift conducted animals to a dangerous situation, in terms of thermal uncomfortable and heat stress. Considering the cardiac frequency of F1 males and Doper female and the respiratory frequency of Dorper males and females and of F1 males, they were more stressed. It demonstrated the reduced capacity of the exotic genotype and their crossbreed for adaptation.

Index terms: Adaptability, heat stress, breed, breathing frequency, temperature rectal.

(Recebido para publicação em 11 de setembro de 2003 e aprovado em 27 de outubro de 2003)

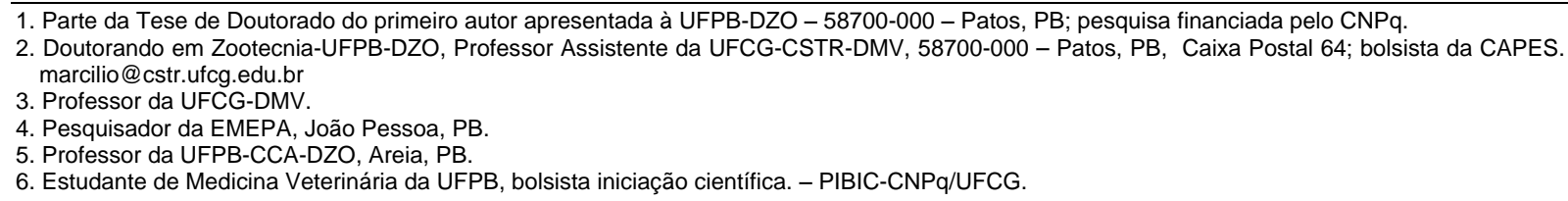




\section{INTRODUÇÃO}

A Região Nordeste do Brasil é a parte mais oriental do continente sul-americano, situada entre as latitudes $3^{\circ}$ e $18^{\circ} \mathrm{S}$ e as longitudes de $35^{\circ}$ e $46^{\circ} \mathrm{W}$. A zona semi-árida nordestina, que corresponde a $74,30 \%$ da superfície do Nordeste, apresenta um clima tropical seco, com uma estação úmida ou chuvosa anual de 4 a 6 meses, seguida por uma estação seca de 6 a 8 meses. A precipitação média anual gira em torno de $700 \mathrm{~mm}$ e a temperatura é alta durante o ano inteiro, com médias térmicas entre $23-28^{\circ} \mathrm{C}$.

O rebanho ovino do Nordeste é representado por um efetivo de aproximadamente 6,7 milhões de cabeças, correspondente a $48,1 \%$ do rebanho nacional (IBGE, 1996), composto em sua vasta maioria por animais deslanados e semilanados, dos quais os crioulos são os seus principais representantes, seguidos pelos animais das raças Santa Inês, Morada Nova e Somalis. Embora numericamente expressivo, esse rebanho apresenta níveis acentuadamente reduzidos de desempenho (GUIMARÃES FILHO et al., 2000). Segundo Sousa e Leite (2000), apesar de as raças ovinas deslanadas apresentarem excelentes qualidades de adaptação e de reprodução, apresentam baixos índices de produtividade, especificamente os relacionados à qualidade de carcaça. A alternativa poderia ser a utilização de ovinos semideslanados, como, por exemplo, a raça Dorper, em cruzamentos planejados com ovelhas do tipo SRD ou mesmo com raças como a Santa Inês.

Por outro lado, para Hopkins et al. (1978), o estresse calórico tem sido reconhecido como um importante fator limitante da produção ovina nos trópicos. Entre a alternativa de adequar as condições ambientais aos animais e a de selecionar animais capazes de produzir satisfatoriamente em ambientes adversos, essa última parece ser a solução mais prática para o momento. Há, portanto, segundo Monty Júnior et al. (1991), a necessidade de se conhecerem a tolerância e a capacidade de adaptação das diversas raças como forma de embasamento técnico à exploração ovina, bem como as propostas de introdução de raças em uma nova região ou mesmo o norteamento de programa de cruzamento, visando à obtenção de tipos ou raças mais adequadas a uma condição específica de ambiente. Segundo Baccari Júnior (1990), as avaliações de adaptabilidade dos animais aos ambientes quentes podem ser realizadas por meio de testes de adaptabilidade fisiológica e de adaptabilidade de rendimento ou produção. Para Bianca e Kunz (1978), a temperatura retal e a frequiência respiratória são consideradas as melhores variáveis fisiológicas para estimar a tolerância de animais ao calor. Recentemente, Santos et al. (2003), estudando as respostas fisiológicas de ovinos Santa Inês, Morada Nova e de seus mestiços com a raça Dorper, concluíram que os animais da raça Morada Nova foram os mais adaptados, enquanto os mestiços Santa Inês x Dorper apresentaram-se como os menos adaptados.

Objetivou-se com este trabalho avaliar a adaptabilidade fisiológica de ovinos das raças Dorper, Santa Inês e seus mestiços (produtos F1) às condições climáticas do curimataú ocidental da região semi-árida paraibana, por meio da temperatura retal, freqüência respiratória, batimentos cardíacos e movimentos ruminais.

\section{MATERIAL E MÉTODOS}

O experimento foi conduzido na Estação Experimental de Pendência, pertencente à Empresa de Pesquisa Agropecuária da Paraíba (EMEPA), localizada no município de Soledade, na região semi-árida do curimataú ocidental paraibano, situada a $7^{\circ} 8^{\prime} 18^{\prime \prime} \mathrm{S}$ e $36^{\circ} 27^{\prime}$ 2"W. Gr. e a uma altitude em torno de $534 \mathrm{~m}$.

Os animais experimentais foram 48 cordeiros, sendo 24 machos e 24 fêmeas, com peso vivo médio de 18-20 kg, distribuídos em 3 grupos genéticos com 16 animais em cada grupo: G1 = animais da raça Santa Inês, $\mathrm{G}_{2}=$ animais da raça Dorper e $\mathrm{G}_{3}=$ animais mestiços meio- sangue $\left(F_{1}\right)$ das raças Dorper x Santa Inês. Esses animais foram mantidos, durante o período experimental, em regime semi-intensivo, sob luminosidade natural, alimentados a pasto, com suplementação concentrada e mineral mais água ad libitum.

As frequiências respiratória e cardíaca e os movimentos ruminais foram aferidos usando-se um estetoscópio veterinário e um cronômetro, de acordo com a método descrito por Baccari (1990). Todos os parâmetros fisiológicos foram medidos duas vezes ao dia, às 9 e às 15 horas, em 2 dias por semana e durante 3 semanas da época mais quente do ano (estação seca do ano). Paralelamente às medições das variáveis fisiológicas, foram registradas a temperatura e a umidade relativa do ar por intermédio de termo-higrômetro digital, a temperatura do globo negro pelo termômetro globo negro de Vernon e a temperatura úmida e seca pelo termômetro de bulbo seco e bulbo úmido. Diariamente, durante todo o período experimental, foram determinadas as temperaturas máxima e mínima. $\mathrm{O}$ índice de temperatura do globo negro e umidade (ITGU) foi determinado conforme metodologia de Buffington et al. (1981).

$\mathrm{O}$ delineamento experimental utilizado foi o inteiramente casualizado com 4 repetições num esquema fatorial de $3 \times 2 \times 2$ (três genótipos, dois sexos 
e dois turnos de coletas). Os resultados obtidos foram submetidos à análise de variância pelo programa SAS INSTITUTE (1996) e os valores médios foram comparados pelo teste de Tukey a $5 \%$ de probabilidade.

\section{RESULTADOS E DISCUSSÃO}

As médias das variáveis ambientais e o Índice de Temperatura do Globo Negro e Umidade (ITGU) durante os dois turnos experimentais encontram-se na Tabela 1.

Segundo o National Weather Service - USA, citado por Baêta (1985), os valores de ITGU até 74, de 74 a 79 , de 79 a 84 e acima de 84 definem situação de con- forto, de alerta, de perigo e de emergência, respectivamente. Pelos dados da Tabela 1, verifica-se que as condições climáticas durante o período experimental levaram os animais à situação de alerta durante o turno da manhã e à situação de perigo térmico durante o turno da tarde, ambas consideradas como de desconforto térmico para os animais.

Das três fontes de variações consideradas, sexo, genótipo e turno, a última exerceu efeito significativo $(\mathrm{P}<0,05)$ em todos os parâmetros fisiológicos analisados, sem interagir com sexo e genótipo (Tabela 2); enquanto o genótipo, por sua vez, interagiu com o sexo $(\mathrm{P}<0,05)$ em relação à freqüência cardíaca e à freqüência respiratória, conforme Tabela 3 .

TABELA 1 - Valores médios da temperatura do bulbo seco (TBS), bulbo úmido (TBU), termômetro de globo negro (TGN), umidade relativa (UR), valores absolutos da temperatura máxima (TMX) e mínima (TMN), temperatura do ponto de orvalho (TPO) e índice de temperatura do globo negro (ITGU) nos turnos da manhã e da tarde e média diária.

\begin{tabular}{|c|c|c|c|c|c|c|c|c|}
\hline \multirow{2}{*}{$\begin{array}{c}\text { Turnos } \\
\text { Experimentais }\end{array}$} & \multicolumn{6}{|c|}{ Temperatura do $\operatorname{Ar}\left({ }^{\circ} \mathbf{C}\right)$} & \multirow{2}{*}{ UR (\%) } & \multirow{2}{*}{ ITGU } \\
\hline & TBS & TBU & TGN & TMX & TMN & TPO & & \\
\hline Manhã & 25,3 & 20,0 & 27,5 & - & - & 18,0 & 63,0 & 75,5 \\
\hline Tarde & 31,2 & 22,0 & 34,6 & - & - & 17,5 & 45,0 & 82,4 \\
\hline Média diária & 28,3 & 21,0 & 31,1 & 33,2 & 23,3 & 17,8 & 54,0 & 79,0 \\
\hline
\end{tabular}

TABELA 2 - Médias da freqüência cardíaca (FC), freqüência respiratória (FR), movimentos ruminais (MR) e temperatura retal (TR) em função do turno.

\begin{tabular}{ccccc}
\hline Turno & FC & FR & MR & TR \\
\hline Manhã & $105,67^{\mathrm{a}}$ & $64,38^{\mathrm{a}}$ & $1,30^{\mathrm{a}}$ & $39,50^{\mathrm{a}}$ \\
Tarde & $115,30^{\mathrm{b}}$ & $96,47^{\mathrm{b}}$ & $1,50^{\mathrm{b}}$ & $40,00^{\mathrm{b}}$ \\
\hline
\end{tabular}

Nas colunas, médias seguidas da mesma letra não diferem significativamente entre si, pelo teste de Tukey a $5 \%$ de probabilidade.

TABELA 3 - Médias da Freqüência Cardíaca (FC) e da Freqüência Respiratória (FR) de ovinos em função da interação entre Genótipo (GENO) e sexo (SEXO).

\begin{tabular}{|c|c|c|c|c|}
\hline \multirow{3}{*}{ GENO } & \multicolumn{2}{|c|}{ FC } & \multicolumn{2}{|c|}{ FR } \\
\hline & \multicolumn{2}{|c|}{ Sexo } & \multicolumn{2}{|c|}{ Sexo } \\
\hline & Macho & Fêmea & Macho & Fêmea \\
\hline Santa Inês & $100,80^{\mathrm{Aa}}$ & $106,60^{\mathrm{Aa}}$ & $52,40^{\mathrm{Aa}}$ & $66,65^{\mathrm{Aa}}$ \\
\hline Mestiço & $120,40^{\mathrm{Ba}}$ & $111,30^{\mathrm{ABa}}$ & $92,00^{\mathrm{Ba}}$ & $70,00^{\mathrm{Aa}}$ \\
\hline Dorper & $108,70^{\mathrm{Aa}}$ & $115,00^{\mathrm{Ba}}$ & $91,30^{\mathrm{Ba}}$ & $110,20^{\mathrm{Ba}}$ \\
\hline
\end{tabular}

Nas colunas, médias seguidas da mesma letra maiúscula, e nas linhas, médias seguidas da mesma letra minúscula não diferem significativamente entre si pelo teste de Tukey a $5 \%$ de probabilidade. 
Nos trópicos, segundo Burgos (1979), a amplitude térmica ao longo do ano é baixa (menor que $5^{\circ} \mathrm{C}$ ), embora a amplitude diária seja elevada (em torno de $10^{\circ} \mathrm{C}$ ). Isso provavelmente explique porque o turno foi, entre os fatores de variação considerados, o mais importante quando da resposta fisiológica do animal diante das condições climáticas, além de demonstrar que o período da tarde causa mais desconforto térmico aos animais do que o período da manhã.

Segundo McLean e Calvert (1972), a evaporação respiratória e cutânea são os mecanismos mais importantes para eliminar o excesso de calor pelos ruminantes. Para Ledezma (1987), a sudorese é menos importante do que a evaporação respiratória para os ovinos e quando esses são expostos a elevadas temperaturas, a taxa respiratória aumenta. Em ambientes termoneutros ou com temperaturas abaixo da termoneutralidade, as evaporações cutânea e respiratória nos ovinos contribuem igualmente com cerca de $25 \%$, ou menos, do total de calor perdido. Todavia, acima da termoneutralidade, as perdas de calor por evaporação aumentam progressivamente para igualar a produção de calor, sendo utilizada principalmente a evaporação respiratória, pois a importância da evaporação cutânea nesses casos é desprezível (JOHNSON, 1976).

Hales e Brown (1974) reportam que a taxa de respiração basal da espécie ovina é cerca de 25 a 30 mov/min (movimentos/minuto), podendo subir, segundo Terrill e Slee (1991), a $300 \mathrm{mov} / \mathrm{min}$ em ovinos estressados. A taxa de respiração pode quantificar a severidade do estresse pelo calor, em que uma freqüência de 40$60,60-80,80-120 \mathrm{mov} / \mathrm{min}$ caracteriza um estresse baixo, médio-alto e alto para os ruminantes, respectivamente; e acima de 150 para bovinos e 200 para ovinos, o estresse é classificado como severo (SILANIKOVE, 2000).

A frequiência respiratória, nesta pesquisa, foi significativamente maior $(\mathrm{P}<0,05)$ durante o turno da tarde $(96,47 \mathrm{mov} / \mathrm{min})$ do que no turno da manhã $(64,38$ mov/min) e com base na classificação Silanikove (2000), pode-se dizer que os ovinos apresentaram estresse térmico alto à tarde e de médio a alto pela manhã.

Os resultados obtidos foram semelhantes a algumas pesquisas com ovinos (SOUZA et al., 1990; SANTOS et al., 2003) e caprinos (BRITO, 1987; MEDEIROS et al., 1998; SILVEIRA et al., 2001). Todavia, vale ressaltar o trabalho de Santos et al. (2003), os quais, sob condições climáticas equivalentes e genótipos similares, obtiveram uma média de freqüência respiratória à tarde de 87,43 , contra $59,13 \mathrm{mov} / \mathrm{min}$ pela manhã. Com esses dados, além de se confirmar a ocorrência de estresse mais elevado no turno mais quente do dia, demonstra-se também que esse era de igual severidade, ou seja, um estresse elevado.

A temperatura corporal é o resultado da diferença entre energia térmica produzida mais a recebida pelo organismo animal e a energia térmica dissipada desse para o meio. De acordo Johnson (1980), a temperatura retal é um indicador dessa diferença e pode ser usada para avaliar a adversidade do ambiente térmico sobre os animais. Os ovinos apresentam uma temperatura retal média de aproximadamente $39,1^{\circ} \mathrm{C}$ (SWENSON, 1988) e, de acordo com McDowell et al. (1976), uma elevação $1{ }^{\circ} \mathrm{C}$ ou menos na temperatura retal é o bastante para reduzir o desempenho na maioria das espécies de animais domésticos.

O turno influenciou de forma significativa $(\mathrm{P}<0,05)$ a temperatura retal, de modo que a temperatura vespertina $\left(40,0^{\circ} \mathrm{C}\right)$ foi superior à temperatura retal matutina $\left(39,5^{\circ} \mathrm{C}\right)$, significando que os animais não foram capazes de dissipar todo o calor necessário para manter sua temperatura corporal dentro do limite basal médio $\left(39,1^{\circ} \mathrm{C}\right)$, principalmente durante o período da tarde. Resultado esse atribuído provavelmente à maior incidência da radiação solar à tarde e confirmado por Santos et al. (2003), ao trabalharem com diversos genótipos, inclusive dois (Santa Inês e mestiços meio-sangue Santa Inês/Dorper) dos três genótipos ovinos utilizados nesta pesquisa, e sob as mesmas condições do trópico semi-árido paraibano, obtiveram uma temperatura retal média de todos os genótipos em torno de $39,5^{\circ} \mathrm{C}$ para o turno da tarde e de $39,3^{\circ} \mathrm{C}$ para o turno da manhã. Por sua vez, Souza et al. (1990) alcançaram para ovinos Santa Inês e Morada Nova e sob mesmas condições climáticas regionais, embora submetendo os animais ao sol e à sombra, uma média aproximada de 38,7 e $38,8^{\circ} \mathrm{C}$ para $\mathrm{o}$ período da manhã e tarde, respectivamente.

Embora a maioria dos pesquisadores afirmem, a exemplo de Bianca e Kunz (1978), que a temperatura retal e a frequiência respiratória são consideradas as melhores variáveis fisiológicas para estimar a tolerância de animais ao calor, foi incluída neste estudo a freqüência dos movimentos ruminais e dos batimentos cardíacos, como forma de aprofundar os conhecimentos acerca do assunto e para acompanhar as tendências de pesquisas mais recentes com outras espécies de animais.

A taxa de movimentos ruminais foi significativamente maior $(\mathrm{P}<0,05)$ durante o turno da tarde $(1,50$ mov./min.), do que no turno da manhã $(1,30$ mov./min.), o que vai de encontro com alguns pesquisadores, os quais em seus trabalhos têm associado o 
estresse térmico com uma redução da motilidade do rúmen-retículo e, conseqüentemente, com um maior tempo de retenção da digesta no rúmen-retículo, uma menor ingestão de alimentos e com uma maior taxa de digestibilidade, especialmente da fibra dietética, pelos ovinos, caprinos e bovinos estressados pelo calor (ATTEBERY e JOHNSON, 1969; BHATTACHARYA e HUSSAIN, 1974; HEAD, 1989; SILANIKOVE, 1992).

A taxa de pulsação dos animais domésticos apresenta grande variação sob diferentes testes de tolerância térmica e entre diferentes grupos genéticos (SINGH e BHATTACHARYYA, 1990), provavelmente porque, segundo Johnson et al. (1991) e Elvinger et al. (1992), o estresse térmico pode causar diluição, concentração ou não ter nenhum efeito sobre o volume do plasma sanguíneo. Todavia, uma freqüência cardíaca reduzida é mais típica em animais sob estresse térmico e está associada com uma taxa reduzida de produção de calor em resposta a temperaturas ambientais elevadas (KADZERE et al., 2002).

A freqüência de batimentos cardíacos registrada foi influenciada significativamente $(\mathrm{P}<0,05)$ pelo turno, sendo a taxa cardíaca vespertina $(115,30$ mov/min) superior à taxa matutina (105,67 mov/min), o que contraria a hipótese de Kadzere et al. (2002), mas não foge das possibilidades relatadas por Johnson et al. (1991) e Elvinger et al. (1992). Esses resultados, embora sejam inferiores aos 126,4 , para o período da manhã, e 133,2 mov/min, para o período da tarde, alcançados por Santos et al. (2003), são concordantes no que tange à superioridade da taxa vespertina sobre a matutina.

Com relação aos genótipos, segundo Pimenta Filho et al. (2000), as raças ovinas naturalizadas do Nordeste brasileiro têm elevado valor adaptativo, enquanto a única raça especializada de corte que deveria ser criada nos trópicos e que poderia ser cruzada com aquelas raças naturalizadas seria a raça Dorper. Segundo fontes da revisão de Cloete et al. (2000), a raça composta Dorper da África do Sul, por resultar do cruzamento da raça Black-Head Persian, de grande rusticidade, com a raça Dorset Horn, de grande capacidade para produção de carne, ela produz satisfatoriamente sob as condições áridas da África do Sul e em virtude de sua rusticidade e adaptabilidade, tem sido muito exportada para outros países, o que tem ocorrido com freqüência na Região Nordeste do Brasil. Embora a raça Dorper seja especializada na produção de carne e tenha sua origem em latitudes maiores e, portanto, possua condições edafoclimáticas menos adversas, porém não muito diferentes daquelas do semi-árido nordestino, inclusive com uma condição de aridez maior; não era de se esperar dessa raça e de suas cruzas resultados de adaptabilidade muito aquém das raças naturalizadas. Com base nesse contexto, o genótipo não influenciou os movimentos ruminais e a temperatura retal, embora tenha tido efeito e interagido $(\mathrm{P}<0,05)$ com o sexo em relação à freqüência cardíaca e à frequiência respiratória (Tabela 3). Provavelmente, os animais de todos os genótipos, inclusive Dorper e mestiços, ao usarem os mecanismos primários de dissipação de calor, ou seja, a evaporação respiratória e a vasodilatação periférica, perderam calor o suficiente para manter sua temperatura corporal dentro dos limites basais, sem a necessidade do uso de outros mecanismos, como a redução da ingestão alimentar decorrente de uma menor motilidade do rúmen-retículo, por exemplo.

Por outro lado, os animais mestiços do sexo masculino apresentaram freqüência cardíaca maior $(\mathrm{P}<0,05)$ que aquela apresentada pelos demais genótipos do mesmo sexo que, por sua vez, não diferiram $(\mathrm{P}>0,05)$ entre si. A frequiência cardíaca das fêmeas Dorper foi significativamente maior que a das Santa Inês, e a das fêmeas Mestiças foram iguais $(\mathrm{P}>0,05)$ as Dorper e Santa Inês (TABELA 3). Quanto à frequiência respiratória, os machos Mestiços e Dorper não diferiram entre si $(\mathrm{P}>0,05)$, mas foram superiores $(\mathrm{P}<0,05)$ aos machos Santa Inês; da mesma forma, as fêmeas Dorper foram inferiores $(\mathrm{P}<0,05)$ às fêmeas Santa Inês e Mestiças, as quais, por sua vez, foram iguais $(\mathrm{P}>0,05)$ entre si (Tabela 3$)$.

Os animais machos da raça Santa Inês apresentaram taxas respiratórias e cardíacas menores do que os machos Mestiços e Dorper, que foram iguais entre si, bem como suas fêmeas tiveram taxas, tanto cardíaca e respiratória, inferiores às fêmeas Dorper e iguais às fêmeas mestiças, provavelmente por causa de sua maior adaptabilidade natural, como era de se esperar em um ambiente no qual a raça Santa Inês teve origem. Os animais mestiços apresentaram taxas, na maioria das vezes, intermediárias às duas raças, provavelmente porque $50 \%$ de sua carga genética é da raça Santa Inês e $50 \%$, da raça Dorper. Nenhuma diferença $(\mathrm{P}>0,05)$ ocorreu entre os sexos, tanto no turno da manhã, quanto no turno da tarde, independentemente do genótipo. Todavia, o sexo interagiu com o genótipo em relação à frequiência respiratória e cardíaca, já discutidos anteriormente.

\section{CONCLUSÕES}

Sob as condições climáticas impostas pelo experimento, o turno da tarde conduziu os animais, independentemente de genótipo e sexo, à situação de perigo e à condição de elevado estresse calórico. Em- 
bora todos os genótipos e sexos tenham mantido sua temperatura retal dentro dos limites basais, a maior freqüência respiratória e cardíaca dos animais Dorper e seus mestiços demonstrou menor grau de adaptabilidade do genótipo exótico. Diante disso, a raça Dorper pode ser utilizada em programas de cruzamentos com o rebanho Santa Inês nativo, desde que medidas de manejo sejam adotadas para minimizar o estresse calórico do turno vespertino.

\section{REFERÊNCIAS BIBLIOGRÁFIAS}

ATTEBERY, J. T.; JOHSON, H. D. Effects of environmental temperature, controlled feeding and fasting on rumen motility. Journal Animal Science, Champaign, v. 29, p. 727-737, 1969.

BACCARI JÚNIOR, F. Métodos e técnicas de avaliação da adaptabilidade dos animais às condições tropicais. In: SIMPÓSIO INTERNACIONAL DE BIOCLIMATOLOGIA ANIMAL NOS TRÓPICOS: PEQUENOS E GRANDES RUMINANTES, 1., 1990, Sobral-CE. Anais... Sobral: EMBRAPA-CNPC, 1990. p. 9-17.

BAÊTA, F. C. Responses of lactating dairy cows to the combined effects of temperature, humidity and wind velocity in the warm season. 1985. $218 \mathrm{f}$. Thesis (Ph.D) - University of Missouri, Missouri, 1985.

BHATTACHARYA, A. N.; HUSSAIN, F. Intake and utilization of nutrients in sheep fed different levels of roughage under heat stress. Journal Animal Science, Champaign, v. 38, p. 877-886, 1974.

BIANCA, W.; KUNZ, P. Physiological reactions of three breedes of goats to cold, heat and hight altitude. Livestock production Science, [S.1.], v. 5, n. 1, p. 5769, 1978.

BRITO, V. F. F. Estudo de caprinos mestiços em ambiente de sol e de sombra, nas condições de viçosa, Minas Gerais. 1987. 47 f. Dissertação (Mestrado) - Universidade Federal de Viçosa, Viçosa, 1987.

BUFFINGTON, D. E.; COLLAZO-AROCHO, A.; CANTON, G. H. Black globe-humidity index (BGHI) as confort equation for dairy cows. Transactions of the ASAE, Michigan, v. 24, n. 3, p. 711-714, 1981.
BURGOS, J. J. Clima tropical y subtropical. In: HELMAN, M. B. Ganaderia Tropical. Buenos Aires: El Ateneo, 1979. p. 1-28.

CLOETE, S. W. P.; SNYMAN, M. A.; HERSELMAN, M. J. Productive performance of Dorper sheep. Small Rumming Research, [S.1.], v. 36, p. 119-135, 2000.

ELVINGER, F.; NATZKE, R.; HANSEN, P. Interactions of heat stress and bovine somatotropin affecting physiology and immunology of lactating cows. Journal Dairy Science, Champaign, v. 75, p. 449-462, 1992.

GUIMARÃES FILHO, C.; SOARES, J. G. G.; ARAÚJO, G. G. L. Sistemas de produção de carnes caprina e ovina no semi-árido nordestino. In: SIMPÓSIO INTERNACIONAL SOBRE CAPRINOS E OVINOS DE CORTE, 1., 2000, João Pessoa, PB. Anais... João Pessoa: EMEPA-PB, 2000. p. 21-34.

HALES, J. R. S.; BROWN, G. D. Net energetic and thermoregulatory efficiency during panting in the sheep. Comp. Biochemical Physiology, [S.1.], v. 49, p. 413422, 1974.

HEAD, H. H. The strategic use of the physiological potential of the dairy cow. In: SIMPÓSIO LEITEIRO NOS TRÓPICOS, 1., 1989, Botucatu. Anais... Botucatu: [s.n.], 1989. p. 38-89.

HOPKINS, P. S.; KNIGHTS, G. I.; LEFEURE, A. S. Studies of the environmental physiology of tropical Merinos. Australian Journal Agriculture Research, East Medelaine, v. 29, n. 1, p. 61-71, 1978.

INSTITUTO BRASILEIRO DE GEOGRAFIA E ESTATÍSTICA. Censo Agropecuário 1995-1996. Rio de Janeiro, 1996.

JOHNSON, H. D. Evaporative temperature regulation in sheep. In: SPB ACADEMY. Progress in Biometeorology: chapter 1, section 5c. [S.1.]: SPB Academy, 1976. p. 140-147.

JOHNSON, H. D. Depressed chemical thermogenesis and hormonal functions in heat. In:

Environmental Physiology: aging, heat, and altitude. Amsterdam: Elsevier, 1980. p. 3-9. 
JOHNSON, H. D.; LI, R.; MANALU, W.; SPENCERJOHNSON, K. J. Effects of somatotropin on milk yield and physiological responses during summer farm and hot laboratory conditions. Journal Dairy Science, Champaign, v. 74, p. 1250-1262, 1991.

KADZERE, M. R.; MURPHY, N.; SILANIKOVE, E.; MALTZ. Heat stress in lactating dairy cows: a review. Livestock Production Science, [S.1.], v. 77, p. 59-91, 2002.

LEDEZMA, J. J. H. Sheep. In: JOHNSON, E. Bioclimatology and the adaptation of livestock. Amsterdam: Elsevier, 1987. p. 169-179.

McDOWELL, R. E.; HOOVEN, N. W.; CAMOENS, J. $\mathrm{K}$. Effects of climate onperformance of Holsteins in first lactation. Journal Dairy Science, Champaign, v. 59, p. 965-973, 1976.

McLEAN, J. A.; CALVERT, D. T. Influence of air humidity on the partition of heat exchanges of cattle. Journal Agricultural Science, Cambridge, v. 78, p. 303, 1972 .

MEDEIROS, L. F. D.; COUTINHO, J. R.; SCHERER. Reações fisiológicas de caprinos de diferentes raças mantidos à sombra, ao sol e em ambiente parcialmente sombreado. In: REUNIÃO ANUAL DA SOCIEDADE BRASILEIRA DE ZOOTECNIA, 35., 1998, Botucatu. Anais... Botucatu: SBZ, 1998. v. 1.

MONTY JÚNIOR, D. E.; KELLY, L. M.; RICE, W. R. Aclimatization of St Croix, Karakul and Rambouillet sheep to intense and dry summer heat. Small Rumming Research, [S.1.], v. 4, n. 4, p. 379392, 1991.

PIMENTA FILHO, E. C.; RIBEIRO, M. N.; SOUZA, W. H. Melhoramento genético de pequenos ruminantes para carne e leite. In: CONGRESSO NORDESTINO DE PRODUÇÃO ANIMAL, 2., 2000, Teresina, PI. Anais... Teresina: SNPA, 2000. p. 107-116.

SANTOS, J. R. S.; SOUZA, B. B.; SOUZA, W. H.; CEZAR, M. F.; TAVARES, G. P. Avaliação da adaptabilidade de ovinos da raça santa inês, morada nova e mestiços de dorper, no semi-árido. In: REUNIÃO ANUAL DA SOCIEDADE BRASILEIRA DE
ZOOTECNIA, 40., 2003, Santa Maria, RS. Anais... Santa Maria: SBZ, 2003. p. 1-5.

SAS INSTITUTE. Statistical Analysis System: user's guide: statistics. Version 6.11. Washington, 1996. $842 \mathrm{p}$.

SILANIKOVE, N. Effects of water scarcity and hot environment on appetite and digestion in ruminants: a review. Livestock Production Science, [S.1.], v. 30, p. 175-194, 1992.

SILANIKOVE, N. Effects of heat stress on the welfare of extensively managed domestic ruminants. Livestock Production Science, [S.1.], v. 67, p. 118,2000

SILVEIRA, J. O. A.; PIMENTA FILHO, E. C.; OLIVEIRA, E. M.; LOPES, W. B. Respostas adaptativas de caprinos das raças Bôer e Anglo-Nubiano às condições do semi-árido brasileiro-frequencia respiratória. In: REUNIÃO ANUAL DA SOCIEDADE BRASILEIRA DE ZOOTECNIA, 38., 2001, Piracicaba, SP. Anais... Piracicaba: SBZ, 2001. p. 14-16.

SINGH, K.; BHATTACHARYYA, N. K. Cardiorespiratory activity in Zebu and their $\mathrm{F}$ crosses with European breeds of dairy cattle at different ambient temperatures. Livestock Production Science, [S.1.], v. 24, p. 119-128, 1990.

SOUSA, W. H.; LEITE, P. R. M. Ovinos de corte: a raça Dorper. João Pessoa: EMEPA-PB, 2000. 76 p.

SOUZA, B. B.; SILVA, A. M.; VIRGINIO, R. S.; GUEDES JÚNIOR, D. B.; AMORIM, F. U. Comportamento fisiológico de ovinos deslanados no semiárido expostos em ambiente sol e em ambiente sombra. Veterinária e Zootecnia, São Paulo, v. 2, p. 1-8, 1990.

SWENSON, M. J. Duke's physiology of domestic animals. 10. ed. Rio de Janeiro: Guanabara, 1988. 799 p.

TERRILL, C. E.; SLEE, J. Breed differences in adaptation of sheep. In: MAIJALA, K. Genetic resources of pigs, sheep and goat. Amsterdam: Elsevier, 1991. p. 195-233. 